\title{
Alteraciones neuropsicológicas asociadas al consumo de la Dextro-metil-anfetamina en adolescentes mexicanos
}

\author{
Charles Ysaacc Da Silva Rodrigues', Paula Alexandra Carvalho de Figueiredo', Paulo Pombo Marques², José Félix \\ Gómez Calderón ${ }^{3}$ \\ I Universidad de Guanajuato, Guanajuato, México \\ 2 CESUN Universidad, México \\ ${ }^{3}$ Centro de Integración Juvenil León, Guanajuato, México
}

\section{RESUMEN}

Introducción: la ingesta de metanfetamina origina una reacción química con la dopamina segregada en las estructuras de la vía mesolímbica, produciendo alteraciones en los procesos conductuales, emocionales y cognitivos. Objetivo: el objetivo de este trabajo fue identificar los cambios neuropsicológicos asociados al consumo de cristal en estudiantes de educación media superior y superior, en fase inicial de tratamiento, con consumos controlados. Método: es un estudio comparativo con la participación de 170 adolescentes evaluados en los ámbitos neuropsicológicos (NEUROPSI), personalidad (NEO-FFI) y regulación emocional (DASS-21). Resultados: la evaluación de las dimensiones cognitivas por el NEUROPSI revelan diferencias estadísticamente significativas entre los dos grupos. Discusión y conclusiones: el consumo de cristal parece producir una serie de daños neuropsicológicos acentuados, como consecuencia de una sintetización menos pura que ataca determinadas regiones y sistemas cerebrales. Los cambios emocionales demuestran tener vínculos con la conducta y a la vez con el aspecto neuropsicológico, generando cambios que llevan a una inadecuación social por parte de los consumidores.

Palabras clave: metanfetamina, cristal, neuropsicología, emoción, adolescentes.

\begin{abstract}
Introduction: the consumption of methamphetamine creates a chemical reaction with the dopamine segregated in the structures of the mesolimbic way, producing alterations in the behavioral emotional and cognitive processes. Objective: the objective of this work was to identify the neuropsychological changes associated with the consumption of crystal in students of medium and high education, in an initial stage of treatment, with controlled consumption. Method: is a comparative study, with the participation of 170 adolescents, evaluated in the neuropsychological (NEUROPSI), personality (NEO-FFI) and emotional regulation (DASS-21) categories. Results: the evaluation of the cognitive dimensions by NEUROPSI reveal differences statistically significant between the two groups. Discussion and conclusion: the consumption of cristal seems to produce a series of acute neuropsychological damages, as a consequence of a sintering less pure that attack to determine regions and cerebral systems. Emotional changes demonstrated to have links with the behaviours and as the same time with the neuropsychological aspect, generating changes that carry a social inadequacy by consumers.
\end{abstract}

Keywords: methamphetamine, crystal, neuropsychology, emotion, teenagers.

\footnotetext{
Autor de correspondencia:

Charles Ysaacc Da Silva Rodrigues, Profesor de Tiempo Completo de la Universidad de Guanajuato. Departamento de Psicología, División de Ciencias de la Salud. Campus León. Blvd. Puente Milenio núm. 1001, Fracción del Predio San Carlos, C.P. 37670, León, Guanajuato. Tel. +52 (477) 2674900 ext. 3637. Correo electrónico: charles.rodrigues@ugto.mx

Recibido: 29 de enero de 2019

Aceptado: 22 de marzo de 2019

doi: 10.28931/riiad.2019.1.03
} 


\section{INTRODUCCIÓN}

La metanfetamina, N-metil-1-fenilpropan-2-amina, es un estimulante psicomotor del Sistema Nervioso Central que funciona en el encéfalo como un agente agonista adrenérgico. Su fórmula empírica es $\mathrm{C}_{10} \mathrm{H}_{15} \mathrm{~N}$, y presenta una alta solubilidad en lípidos, lo que independientemente de la permeabilidad de la barrera hematoencefálica, le permite una fácil transposición al tejido nervioso de la estructura cerebral. Al igual que la metanfetamina, el cristal resulta de la manipulación molecular de la anfetamina hacia la dextro-metil-anfetamina, convirtiéndose en una de las drogas más consumidas en Estados Unidos y México, además del fuerte incremento de consumo en Canadá (Bernardi, 2015; Lu, Kim, Greig, \& Luo, 2017; Müller \& Kornhuber, 2017).

La síntesis del cristal es bastante simple, considerando que tiene como base química la efedrina o la pseudoefedrina, comúnmente encontrados en una variedad de medicamentos, como antigripales y descongestionantes nasales, que no necesitan prescripción médica. Los demás componentes, por lo general, son productos bastante corrosivos y tóxicos, que se mezclan de diferentes maneras, formando componentes activos similares, pero no iguales. Su absorción se realiza por vía del tracto gastrointestinal y, dependiendo de su estado, puede llevar entre tres y seis horas tras su ingestión; su vida media en el organismo es entre nueve y 12 horas y se excreta casi en su totalidad por vía urinaria (Shokoohi et al., 2018; Wu et al., 2018).

De acuerdo con del grado de pureza y concentración tras su sinterización, su consumo puede generar sensación de euforia y mejoramiento del estado de ánimo, aumento de la energía, actividad física, la libido y la capacidad sexual, principalmente en mujeres, así como disminución de la fatiga y la necesidad de dormir. En el caso de la metanfetamina de muy buena calidad, el individuo percibe un incremento en el rendimiento de tareas mentales y procesos neurocognitivos, como la atención y memoria. Entre sus efectos adversos más comunes se encuentran: aumento de la presión sanguínea, sudoración, temblores, náuseas, diarrea, taquicardia, convulsiones, sensación persecutoria y celotipia (Alexander et al., 2017; Da Silva, 2018).

El consumo de cristal provoca una liberación significativa de neurotransmisores monoaminérgicos, entre ellos la dopamina, que se distribuye por las vías específicas (mesocortical, nigroestriatal y tuberoinfundibular). En el caso de la ingestión de estimulantes psicomotores, la vía dopaminérgica de activación más relevante es la mesolímbica, la cual representa la conexión mesencéfalo-sistema límbico, y es responsable de una serie de conexiones de fibras nerviosas entre estructuras, enfati- zando: la sustancia negra y los núcleos de rafe en el mesencéfalo; y de los ganglios basales, corteza cingulada, cuerpos mamilares, septum, hipocampo y amígdala, en el sistema límbico (Brooks et al., 2017; Oh et al., 2016).

Una vez ingerida la dextro-metil-anfetamina, produce una reacción química con la dopamina segregada en las estructuras de la vía mesolímbica, generando alteraciones en los procesos conductuales, emocionales y cognitivos. Aunque no exista un patrón comportamental sistematizado del consumo de cristal, sería esperado, con base en el aspecto anatomo-funcional, que se produjeran dificultades agravadas en la identificación de situaciones de alerta, la planificación motora de movimientos, el control de respuesta motriz a estímulos emocionales, el reconocimiento de las habilidades sociales, la comunicación de alto orden y el control de los ciclos de sueño (Da Silva, 2018; Lindsley \& Hopkins, 2017; Müller \& Kornhuber, 2017).

A nivel emocional se pueden encontrar dos importantes tipos de proyecciones nerviosas asociadas con el núcleo accumbens: las proyecciones límbicas, inscritas a las limitaciones en el reconocimiento de emociones, proceso de empatía, hiperactivación del deseo sexual y activación del círculo de odio ubicado en el putamen (que se encarga de generar sentimientos como ira, miedo, temor, entre otras); y las proyecciones extra límbicas, relacionadas, por un lado, con estructuras filogenéticamente más primitivas que generan la sensación de deseo adictivo incontrolable y, por otro lado, asociadas con los núcleos de rafe que transmiten ansiedad, angustia y agresividad en el proceso inicial de la abstinencia (Lu et al., 2017; Shokoohi et al., 2017).

En el ámbito cognitivo debe considerarse una diversidad de interacciones bioquímicas que resultan del procesamiento de la información sensoriomotora del mesencéfalo, la integración de los estados emocionales originados por los sistemas límbico y de recompensa y la consolidación de esta información en la neocorteza, a partir de procesos como: pensamiento, razonamiento, aprendizaje, consolidación de memoria, evaluación del entorno, resolución de problemas, control de respuestas emocionales, interpretación de expresiones faciales y respuesta conductual apropiada, entre otros aspectos, que debido a la ingesta de la dextro-metil-anfetamina se verían seriamente alterados (Da Silva, 2018; Wu et al., 2018).

La investigación sobre los cambios derivados del consumo de dextro-metil-anfetamina se vuelve necesaria considerando los aspectos anteriormente mencionados, así como el aumento de diversos problemas entre los consumidores como: el incremento de suicidios en consumidores de cristal, debido a los cambios bioquímicos, anatómicos y endócrinos producidos por la sustancia; el nacimiento de niños con problemas orgánicos 
adquiridos como resultado de deformaciones del tubo neural; los riesgos de enfermedades asociadas a la lactancia cuando las progenitoras son consumidoras; la proliferación de enfermedades sexualmente transmisibles, teniendo en cuenta la hiperactivación del deseo sexual; y la posibilidad de enfermedades psicopatológicas inducidas por cambios anatomo-estructurales de los sistemas responsables por las conductas y emociones (Bernardi, 2015; Lu et al., 2017; Müller \& Kornhuber, 2017).

Asimismo, el consumo de cristal aumenta desmesuradamente debido a su bajo costo comercial, aunque para asegurar esa condición, la dextro-metil-anfetamina se produce con componentes de menor calidad, cada vez más neurotóxicos. En cuanto al perfil del consumidor, se ha ajustado a los nuevos roles sociales, en los cuales los más jóvenes pasaron a ser los principales clientes ("Müller \& Kornhuber, 2017; Wu et al., 2018).

El consumo de cristal entre los jóvenes genera especial preocupación, fundamentalmente porque se encuentran en una fase de desarrollo encefálico que acarrea una serie de alteraciones morfológicas, fisiológicas, bioquímicas y psicológicas. En este sentido, el objetivo de esta investigación se orientó hacia la identificación de las alteraciones neuropsicológicas asociadas al consumo de cristal, en estudiantes de educación media superior y superior, en fase inicial de tratamiento, con consumos controlados. Se esperó encontrar alteraciones al nivel mesolímbico y del núcleo accumbens, debido a sus conexiones corticales y subcorticales con la corteza y el sistema límbico, proporcionando un respaldo en la explicación de dichos cambios.

\section{MÉTODO}

\section{Diseño}

Esta investigación es un estudio descriptivo y comparativo que busca conocer las consecuencias del consumo de cristal, con un enfoque cuantitativo, a través de medición de variables independientes.

\section{Participantes}

La muestra de este trabajo fue de 170 adolescentes mexicanos estudiantes de educación media superior y superior, 84 mujeres $(49.4 \%)$ y 86 varones (50.6\%). La edad media de los participantes fue de 19 años y fueron divididos homogéneamente en dos grupos: un grupo de usuarios (G1) y un grupo de no usuarios (G2). Todos los participantes eran estudiantes de alguna preparatoria o universidad.

El G1 fue constituido por 82 adolescentes mexicanos de educación media superior y superior, que ade- más se encontraban en fase inicial de tratamiento en una institución ambulatoria mexicana de atención de las adicciones, con consumos controlados, que fueron escogidos por conveniencia. Los participantes tenían una media de edad de 17 años que variaba entre los 16 y los 21 años, su división por género estaba representado por 43 mujeres (52.4\%) y 39 varones (47.6\%).

El G2 fue compuesto por otros 88 adolescentes mexicanos de educación media superior y superior, igualmente elegidos por conveniencia de diversas instituciones de enseñanza en México. Tenían una media de edad de 18 años, que variaba entre los 16 y los 23 años, su división por género representaba 41 mujeres (46.6\%) y 47 varones $(53.4 \%)$.

\section{Instrumentos}

Para la evaluación de los procesos neuropsicológicos se utilizó el NEUROPSI, una prueba que permite el reconocimiento de alteraciones en el funcionamiento cognoscitivo en nueve diferentes dimensiones: orientación, atención y concentración, memoria, lenguaje, lectura, escritura, cálculo, funciones ejecutivas y procesos visoconstructivos, las cuales implican procesos como anticipación, selección de meta, capacidad de secuenciar, planear y organizar la conducta. La prueba está validada para población hispano hablante con una confiabilidad de 0.87 y una validez de 95\%, su aplicación demora entre 25 y 30 minutos, y debe ser realizada en individuos entre 16 y 85 años (Ostrosky, Ardila, \& Rosselli, 2013; Ostrosky, Gomez, Matute, Rosselli, Ardila, \& Pineda, 2019).

La evaluación de personalidad se realizó a través del inventario de personalidad NEO-FFI, en su versión reducida de 60 ítems y cinco dimensiones o rasgos: neuroticismo, extroversión, apertura a la experiencia, amabilidad y responsabilidad. La validación del inventario presenta un nivel de homogeneidad de 0.22 a 0.45 y una consistencia interna de .70 a .71 , mientras que el coeficiente Alfa de Cronbach presenta una fiabilidad de .73 para el rasgo de neuroticismo, .78 para extroversión, .79 para apertura a la experiencia, .70 para amabilidad y .74 para responsabilidad (Meda-Lara, Moreno-Jiménez, García, Palomera Chávez, \& Mariscal Santiago, 2015; Widiger \& Costa, 2013).

En el ámbito emocional se aplicó la versión abreviada de la Escala de Depresión, Ansiedad y Estrés (EDAE), conocida en su versión original como Depression, Anxiety and Stress Scale (DASS), y su versión reducida por DASS-21. Es una escala de autorreporte que incorpora tres dimensiones o subescalas, con siete ítems cada una, que miden la intensidad de estados afectivos de depresión, ansiedad y estrés. En la población mexica- 
na presenta una fiabilidad de 0.81 para la subescala de depresión, de 0.86 para la subescala de ansiedad, y de 0.79 para la subescala de estrés, presentando un Alpha de Cronbach de 0.80 para toda la escala (Gurrola Peña, Balcázar Nava, Bonilla Muños, \& Virseda Heras 2006; Lovibond \& Lovibond, 1995; Román, Santibáñez, \& Vinet, 2016).

\section{Procedimiento}

En el primer contacto efectuado con los participantes, se les explicaron las condiciones de formar parte de este estudio. Además, se recopilaron autorizaciones y consentimientos informados de las instituciones involucradas, y se realizaron historias clínicas de los participantes. Todo el proceso de evaluación fue efectuado en las instalaciones de la institución ambulatoria mexicana para las adicciones, que por cuestiones de anonimato no se identificará.

Se condujeron otras tres sesiones por cada adolescente, de las cuales dos fueron utilizadas para la aplicación de pruebas, y la última se utilizó para la verificación conjunta de expedientes, así como la entrega a los participantes de los resultados de las pruebas.

\section{Consideraciones éticas}

Los participantes fueron informados sobre la investigación, su participación fue voluntaria, en concordancia con los criterios éticos de confidencialidad y anonimato, así como los criterios metodológicos difundidos por la American Psychological Association (APA) y la Ley General de Salud en México.

\section{RESULTADOS}

El análisis de los resultados se realizó a través del Statistical Package for the Social Sciences (SPSS), versión 22 para sistema Windows 10. Las distribuciones aplicadas se realizaron por categorías, agrupando las cualidades de la población estudiada. Se realizó la prueba t para grupos independientes: G1 y G2, con base en una correlación de Pearson del 95\% de confianza.

En un análisis general de los participantes se puede mencionar que en cuanto al nivel de la escolaridad, la muestra se distribuyó en un $47.4 \%$ que estudia en nivel preparatoria; y un $52.6 \%$ que estudia a nivel licenciatura. Dentro del G1 se analizó que, además del cristal, $20.2 \%$ consumen tabaco y mariguana, $11 \%$ combinan los solventes, mariguana, alcohol, piedra, tabaco y posibles hongos, con el cristal; $3 \%$ con solventes, marigua- na, alcohol y piedra; $2.5 \%$ con el alcohol; y $2 \%$ con mariguana, tabaco y cocaína.

Igualmente, se analizó el consumo de sustancias aprobadas para terapéuticas médicas: $84 \%$ de los participantes consumían estas sustancias; $75.2 \%$ no sabe qué tipo de medicamento toma; $2.7 \%$ toma antidepresivos de tipo fluoxetina e imipramina; $2.6 \%$ toma gabapentina, utilizado en el tratamiento del dolor; $1.3 \%$ toma benzodiazepinas, principalmente alprazolan; $1.2 \%$ toma algún tipo de vitaminas y $1 \%$ toma valproato de magnesio, medicamento antiepiléptico y estabilizador del humor.

Frente a un análisis general del NEUROPSI (Tabla 1), se presenta por área neuropsicológica cada una de las significancias estadísticas, esto debido a la extrema relevancia que estos datos presentan. En este sentido, en la Tabla 2 se presentan los resultados de ejecución de tareas evaluadas por la dimensión de orientación en las diferencias estadísticamente significativas que se encontraron entre el G2 y el G1 ( $t=7.82, p=.000)$. Es importante referir que se encontró igualmente significancia en su relación con el estrés ( $r=.138, p=.000)$, sin que existiera relevancia estadística en su relación con la personalidad (Tabla 4).

Las tareas dispuestas en la dimensión de concentración y atención también tuvieron diferencias estadísticamente significativas entre G1 y G2 ( $t=12.205, p=$ .000; Tabla 2); además, presentaron significancia en el estrés $(r=.092, p=.000)$ y ansiedad $(r=.274, p=.000)$, pero sin ninguna relación estadísticamente importante con la personalidad (Tabla 4).

Con relación a las tareas dispuestas en la dimensión de memoria se presentan igualmente diferencias estadísticamente significativas entre los G1 y G2 ( $t=110.70$, $p=.000$; Tabla 2), considerando también significancia para el estrés $(r=.186, p=.000)$ y ansiedad $(r=.286$, $p=.000)$ y sin ninguna relevancia para la personalidad (Tabla 4).

Dentro de la prueba de comparación de medias, la dimensión de lenguaje también tuvo diferencias estadísticamente significativas entre G1 y G2 de la investigación ( $t=70.208, p=.000$; Tabla 2). Asimismo, demostró significancia para las alteraciones en el estrés $(r=.275, p=.000)$ y la ansiedad $(r=.142, p=.000$; Tabla 4).

En relación con las especificidades del NEUROPSI, particularmente la dimensión de funciones ejecutivas, de igual manera se presentan diferencias estadísticamente significativas entre el G1 y G2, ( $t=137.938, p=.000$; Tabla 2). Con todo, en las funciones ejecutivas se presentó significancia para el estrés $(r=.063, p=.000)$, ansiedad $(r=.068, p=.000)$ y depresión $(r=.178, p=.000$; Tabla $4)$. En cuanto a la personalidad, en ningún momento se 
mostró significancia en las relaciones con las funciones neuropsicológicas, lo que igual sería esperado, considerando que existen alteraciones moderadas y severas en la funcionalidad, pero ningún tipo de alteración mental que justificara los cambios conductuales del patrón de la personalidad de estos individuos.

Aunque no se hayan registrado resultados significativos entre funcionalidad neuropsicológica y personalidad, sí existe relevancia estadística entre los resultados de la personalidad y la regulación emocional. Las correlaciones encontradas entre el NEO-FFI y la DASS-21 muestran que existe significancia estadística entre las variables, la subescala de depresión presenta relación con el neuroticismo de $r=.383, p=.000$, con la extraversión de $r=-.114, p=.023$, con la apertura de $r=-.219$, $p=.000$, con la amabilidad de $r=-.220, p=.000$ y con la responsabilidad de $r=-.237, p=.000$. La ansiedad presentó relaciones estadísticamente significativas con el neuroticismo de $r=.435, p=.000$, con la extraversión de $r=-.112, p=.025$, con la apertura de $r=-.336, p=.000$, con la amabilidad de $r=-.263, p=.000$ y con la responsabilidad de $r=-.276, p=.000$. En cuanto al estrés, una significancia de $r=.429, p=.000$ con el neuroticismo, de $r=-.203, p=.000$ con la extroversión, de $r=-.322$, $p=.000$ con la apertura, de $r=-.281, p=.000$ con la amabilidad y de $r=-.301, p=.000$ con la responsabilidad (Tabla 3).

\section{DISCUSIÓN}

Como se refiere inicialmente, la dopamina es un importante componente de la vía mesolímbica que se puede encontrar en la sustancia negra y área retrorrubral del cuerpo estriado, ambas estructuras asociadas a la actividad motora de los ganglios basales; y en las células del área tegmental ventral del mesencéfalo, que participan en el sistema mesolímbico, que se extiende al núcleo accumbens, participando en la recompensa y en el reforzamiento; y el mesocortical, que se proyecta a la corteza prefrontal, donde actúa en apoyo de la actividad neurocognitiva (Figura 1). Esta relación mesocortical es efectivamente la condición anatomo-funcional para una alteración tan efectiva en el ámbito neuropsicológico (Müller \& Kornhuber, 2017; Wu et al., 2018).

Los resultados de esta investigación son muy contundentes en cuanto a las alteraciones en la orientación, concentración y atención, memoria, lenguaje y funciones ejecutivas, que además se presentan como moderadas y graves en algunos casos. Asimismo, no se encontraron cambios al nivel de la personalidad, pero sí se observan importantes alteraciones conductuales, considerando que los resultados apuntan a determinadas regiones cerebrales que nos permiten indagar sobre esas mismas conductas. Así, el núcleo accumbens, además de formar parte del sistema mesolímbico (Figura 1), está asociado al septo y por consiguiente con el sistema límbico, lo que permite su interacción con la corteza cerebral (Bernardi, 2015; Lu et al., 2017; Müller \& Kornhuber, 2017).

El estado comunicativo del núcleo accumbens con el septo se relaciona con aspectos bioquímicos en los cuales participan la dopamina, la serotonina y el glutamato. El septo es relevante por proporcionar comunicación con el hipotálamo y el hipocampo, y por ser un referente para la memoria, atención selectiva y motivación, además de ser regulador de los niveles emocionales y de alerta. Esta comunicación interneuronal de las estructuras referidas, que se realiza por las catecolaminas, apoya las alteraciones neurocognitivas de la atención, memoria e inclusivamente de las funciones ejecutivas, pero sobre todo, presentan base teórica para los cambios motivacionales y emocionales de los consumidores de cristal (Da Silva, 2018; Wu et al., 2018).

El núcleo accumbens tiene, de igual manera, conexiones con la corteza cerebral y con el sistema límbico, por vía de sus receptores de dopamina, serotonina y glutamato. Estas aferencias y eferencias estructurales son responsables del funcionamiento y las alteraciones de las funciones ejecutivas, por un lado, porque se encargan de la información aferente hacia el tálamo y núcleo accumbens, y por otro lado, por sus relaciones con los ganglios basales, la sustancia negra y la corteza motora del lóbulo frontal, que en su conjunto activan el ámbito motriz al momento que sea necesaria la realización de acciones con un contenido emocional dirigidas a lograr una meta específica (Lu et al., 2017; Shokoohi et al., 2018).

Respecto a los núcleos basales, accumbens respeta una serie de funciones mesolímbicas que se distinguen en dos perspectivas: la motivacional, con especificidades de satisfacción en las necesidades más básicas, como comer, beber o tener relaciones sexuales, incidiendo en la novedad o en la privación; por ejemplo, si es una comida diferente y que se prueba por primera vez, o si es algún alimento que durante mucho tiempo estuvo privado por una dieta o por indicación médica; y la motora, que considera lo anterior como parte de la ingesta y conducta social, además de activarse en la recompensa, en administración de drogas y respuestas al estrés ( $\mathrm{Da}$ Silva, 2018; Lindsley \& Hopkins, 2017; Müller \& Kornhuber, 2017).

El conjunto de estructuras asociadas al sistema mesolímbico de la vía dopaminérgica, en conjunto con el núcleo accumbens, protagonizan casi todos los cambios al nivel neuropsicológico, aunque parece fundamental la relación adictiva al mismo núcleo accumbens. Éste se divide anatómicamente en core y shell. El core, 
Tabla 1

Análisis estadístico de la prueba Neuropsicológica NEUROPSI

\begin{tabular}{|c|c|c|c|c|c|c|}
\hline & \multicolumn{2}{|c|}{ Media } & \multicolumn{2}{|c|}{ Desviación Estándar } & \multicolumn{2}{|c|}{ Medida de Error Estándar } \\
\hline & G2 & G1 & G2 & G1 & $\mathrm{G} 2$ & G1 \\
\hline Orientación & -0.135 & -1.101 & 0.62347 & 1.62678 & 0.04409 & 0.11532 \\
\hline $\begin{array}{l}\text { Concentración y } \\
\text { Atención }\end{array}$ & -0.19 & -1.699 & 0.62919 & 1.62666 & 0.04449 & 0.11531 \\
\hline Memoria & -2.74 & -6.392 & 1.71379 & 3.79755 & 0.12118 & 0.2692 \\
\hline Lenguaje & -1.02 & -4.307 & 1.59761 & 3.03373 & 0.11297 & 0.21506 \\
\hline $\begin{array}{l}\text { Funciones } \\
\text { Ejecutivas }\end{array}$ & -1.585 & -8.246 & 1.65726 & 4.72311 & 0.11719 & 0.33481 \\
\hline
\end{tabular}

Nota: G1 = Grupo de Usuarios; G2 = Grupo de No Usuarios.

Tabla 2

Pruebas independientes para el ámbito neuropsicológico

\begin{tabular}{|c|c|c|c|c|c|c|c|c|c|}
\hline & \multicolumn{5}{|l|}{$\begin{array}{c}\text { Prueba de } \\
\text { Levene }\end{array}$} & \multicolumn{4}{|c|}{ Prueba $t$ de Student } \\
\hline & \multirow{2}{*}{$F$} & \multirow{2}{*}{ Sig. } & \multirow{2}{*}{$t$} & \multirow{2}{*}{$\mathrm{Gl}$} & \multirow{2}{*}{$\begin{array}{l}\text { Sig. } \\
\text { Bil. }\end{array}$} & \multirow{2}{*}{$\begin{array}{l}\text { Desviación } \\
\text { Estándar }\end{array}$} & \multirow{2}{*}{$\begin{array}{c}\text { Error } \\
\text { Estándar }\end{array}$} & \multicolumn{2}{|c|}{ Intervalo de Confianza 95\% } \\
\hline & & & & & & & & Inferior & Superior \\
\hline \multirow{2}{*}{ Orientación } & *349.19 & 0 & 7.835 & 397 & 0 & 0.9655 & 0.12323 & 0.72324 & 1.20776 \\
\hline & ** & & 7.82 & 254.692 & 0 & 0.9655 & 0.12346 & 0.72237 & 1.20863 \\
\hline \multirow{2}{*}{$\begin{array}{l}\text { Concentración } \\
\text { y Atención }\end{array}$} & *126.135 & 0 & 12.228 & 397 & 0 & 1.50849 & 0.12337 & 1.26596 & 1.75103 \\
\hline & $\star \star$ & & 12.205 & 255.70 & 0 & 1.50849 & 0.1236 & 1.2651 & 1.75189 \\
\hline \multirow{2}{*}{ Memoria } & *110.70 & 0 & 12.39 & 397 & 0 & 3.65196 & 0.29473 & 3.07253 & 4.23138 \\
\hline & ** & & 12.37 & 275.136 & 0 & 3.65196 & 0.29522 & 3.07078 & 4.23314 \\
\hline \multirow{2}{*}{ Lenguaje } & *70.208 & 0 & 13.548 & 397 & 0 & 3.28653 & 0.24258 & 2.80964 & 3.76343 \\
\hline & $\star \star$ & & 13.529 & 299.646 & 0 & 3.28653 & 0.24292 & 2.80848 & 3.76458 \\
\hline \multirow{2}{*}{$\begin{array}{l}\text { Funciones } \\
\text { Ejecutivas }\end{array}$} & *137.938 & 0 & 18.815 & 397 & 0 & 6.66123 & 0.35403 & 5.96522 & 7.35724 \\
\hline & $\star *$ & & 18.778 & 245.81 & 0 & 6.66123 & 0.35473 & 5.96254 & 7.35992 \\
\hline
\end{tabular}

Nota: $\mathrm{F}$ = valor de la prueba; Sig. = significancia; $\mathrm{t}$ = valor de la prueba $\mathrm{t}$-student; $\mathrm{Gl}$ = grados de libertad; Sig. Bil. = significancia bilateral; ${ }^{\star}$ Se asumen varianzas iguales; **No se asumen varianzas iguales.

Tabla 3

Correlaciones entre el Inventario NEO-FFI y la Escala DASS-21

\begin{tabular}{|c|c|c|c|c|c|c|}
\hline & & Neuroticismo & Extraversión & Apertura & Amabilidad & Responsabilidad \\
\hline \multirow{2}{*}{ Depresión } & Correlación de Pearson & $.383^{* *}$ & $-.114^{*}$ & $-.219^{* \star}$ & $-.220^{\star \star}$ & $-.237^{\star *}$ \\
\hline & Sig. (bilateral) & .000 & .023 & .000 & .000 & .000 \\
\hline \multirow{2}{*}{ Ansiedad } & Correlación de Pearson & $.435^{\star *}$ & $-.112^{*}$ & $-.336^{\star *}$ & $-.263^{\star \star}$ & $-.276^{\star *}$ \\
\hline & Sig. (bilateral) & .000 & .025 & .000 & .000 & .000 \\
\hline \multirow{2}{*}{ Estrés } & Correlación de Pearson & $.429^{* *}$ & $-.203^{\star *}$ & $-.322^{\star *}$ & $-.281^{\star \star}$ & $-.301^{* *}$ \\
\hline & Sig. (bilateral) & .000 & .000 & .000 & .000 & .000 \\
\hline
\end{tabular}

Nota: Sig. (bilateral) = significancia bilateral; * La correlación es significativa en el nivel 0,05 (bilateral); ** La correlación es significativa en el nivel 0,01 (bilateral). 
Tabla 4

Correlaciones entre la prueba NEUROPSI y la Escala DASS-21

\begin{tabular}{|c|c|c|c|c|c|}
\hline & & & Depresión & Ansiedad & Estrés \\
\hline \multirow{10}{*}{ NEUROPSI } & \multirow{2}{*}{ Orientación } & Coeficiente de correlación & -.053 & -.053 & $.138^{*}$ \\
\hline & & Sig. (bilateral) & .457 & .457 & .000 \\
\hline & \multirow{2}{*}{ Concentración y atención } & Coeficiente de correlación & -.078 & $.274^{\star \star}$ & $-.092^{\star}$ \\
\hline & & Sig. (bilateral) & .274 & .000 & .000 \\
\hline & \multirow{2}{*}{ Memoria } & Coeficiente de correlación & -.094 & $.286^{\star *}$ & $.186^{\star}$ \\
\hline & & Sig. (bilateral) & .188 & .000 & .000 \\
\hline & \multirow{2}{*}{ Lenguaje } & Coeficiente de correlación & -.097 & $.142^{*}$ & $.275^{\star \star}$ \\
\hline & & Sig. (bilateral) & .432 & .000 & .000 \\
\hline & \multirow{2}{*}{ Funciones Ejecutivas } & Coeficiente de correlación & $.178^{*}$ & $.068^{*}$ & $.063^{*}$ \\
\hline & & Sig. (bilateral) & .000 & .000 & .000 \\
\hline
\end{tabular}

Nota: Sig. $\left(\right.$ bilateral) $=$ significancia bilateral; * La correlación es significativa en el nivel 0,05 (bilateral); ${ }^{* *}$ La correlación es significativa en el nivel 0,01 (bilateral).

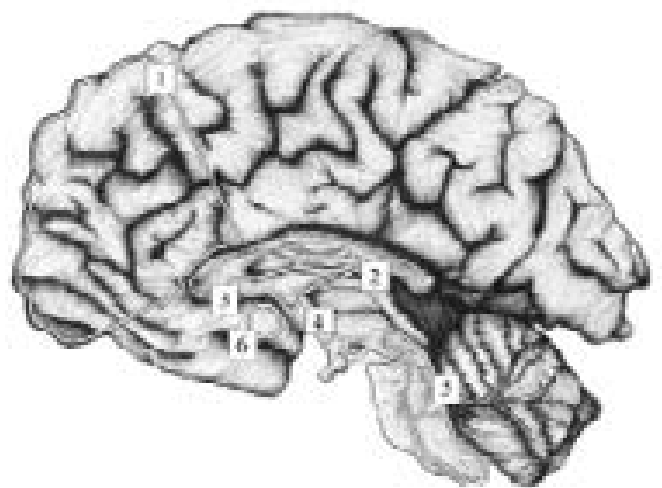

Figura 1. Corte lateral de la sección sagital del cerebro Copyright: Dr. Charles Y. Da Silva Rodrigues

\author{
Leyenda de la figura: \\ 1. Cortera prefrontal. \\ 2. Cuerpo estriado. \\ 3 Sepuim \\ 4. Cuerpo mamular. \\ 5. Núcleos de rafé. \\ 6. Nícleo accunbens.
}

con sus conexiones a la sustancia negra y al globo pálido ventral, más concretamente con los bucles motores cortico-estriado-pálido-talámico, a partir de sus aferencias dopaminérgicas y glutaminérgicas, se vincula con las funciones motrices a partir del nivel emocional, considerando los movimientos, su agilidad y velocidad; mientras que el shell recibe esa neuromodulación dopaminérgica desde el área tegmental (Brooks et al., 2017; Oh et al., 2016).

El núcleo accumbens recibe neurotransmisores moduladores de serotonina desde el núcleo de rafe, y sus amplias conexiones permiten diferentes tipos de aprendizajes, por reforzamiento y castigo es uno de ellos, implicando en el proceso a la memoria, a través de la repetición mental de experiencias agradables, y evitando las representaciones adversas, esto cuando no existen consumos adictivos de cristal. Dentro de este mismo circuito de recompensa se debe considerar el amor y el apego, cuando en las relaciones afectivas el núcleo accumbens interviene en la creación de vínculos tanto físicos como psicológicos en conexiones límbicas y extra límbicas (Alexander et al., 2017; Da Silva, 2018).

En las adicciones, el núcleo accumbens se llena de dopamina cuando existe una sustancia adictiva, así se establece la relación entre la sustancia y el placer, dejando a este mismo núcleo la función del impulso para la acción placentera, debido a sus conexiones con la corteza prefrontal. Así, se entiende la preocupación de que los consumidores abusivos de cristal, por razones obvias, pierdan determinadas capacidades neuropsicológicas, las cuales les dificultará tener una vida estable, tanto social y amorosa, como laboral, aunque una de las más importantes preocupaciones se relaciona con el suicidio. 


\section{CONCLUSIONES}

Con este trabajo se concluye que el consumo de cristal en jóvenes puede producir una serie de daños neuropsicológicos acentuados, al parecer por una sinterización menos pura que ataca determinadas regiones y sistemas cerebrales. Los cambios emocionales demuestran tener vínculos con la conducta y a la vez con el aspecto neuropsicológico, generando cambios que llevan a una inadecuación social, por parte de estos consumidores. Con los resultados arrojados por la evaluación se puede indagar sobre el papel fundamental del sistema mesolímbico y núcleo accumbens en esa desadaptación.

Los cambios de las vías dopaminérgica en el sistema mesolímbico y núcleo accumbens parecen ser los responsables por todas las alteraciones evaluadas en los jóvenes consumidores de dextro-metil-anfetamina. Además del riesgo de suicidio y de problemáticas relacionadas con las madres que consumen embarazadas o cuando amamantan, es importante referir que los daños de esta droga son estructurales, lo que quiere decir que difícilmente se pueden revertir. En muchas situaciones los consumidores son portadores de patología dual, lo que en la práctica complicará más el panorama, y muchas veces, además del consumo, acaban por ser portadores de enfermedades sexualmente transmisibles.

\section{FUENTE DE FINANCIAMIENTO}

La presente investigación fue financiada con recursos propios.

\section{CONFLICTOS DE INTERÉS}

No existen conflictos de interés con relación a los datos presentados.

\section{REFERENCIAS}

Alexander, P. D., Gicas, K. M., Willi, T. S., Kim, C. N., Boyeva, V., Procyshyn, R. M., ... Barr, A. M. (2017). A comparison of psychotic symptoms in subjects with methamphetamine versus cocaine dependence. Psychopharmacology, 234(9-10), 15351547. doi: 10.1007/s00213-017-4551-7

Bernardi, R. E. (2015). Advances in nicotine research in Addiction Biology. Addiction Biology, 20(5), 877-889. doi: 10.1111/ adb.12269

Brooks, S. J., Wiemerslage, L., Burch, K. H., Maiorana, S. A., Cocolas, E., Schiöth, H. B., ... Stein, D. J. (2017). The impact of cognitive training in substance use disorder: the effect of working memory training on impulse control in methamphetamine users. Psychopharmacology, 234(12), 1911-1921. doi: 10.1007/s00213 -017-4597-6
Da Silva R., C. Y. (2018). Neuropsicología del Envejecimiento. México: Manual Moderno. ISBN: 978-607-448-689-6

Gurrola Peña, G. M., Balcázar Nava, P., Bonilla Muños, M. P., Virseda Heras, J. A. (2006) Estructura factorial y consistencia interna de la escala de Depresión Ansiedad y Estrés (DASS-21) en una muestra no clínica. Psicología y Ciencia Social, 8(2), 3-7.

Lindsley, C. W., \& Hopkins, C. R. (2017). Return of D4 Dopamine Receptor Antagonists in Drug Discovery. Journal of Medicinal Chemistry, 60(17), 7233-7243. doi: 10.1021/acs.jmedchem.7b00151

Lovibond, S. H., \& Lovibond, P. F. (1995). Manual for the Depression Anxiety Stress Scales. 2nd. ed. Sydney: Psychology Foundation of Australia.

Lu, T., Kim, P., Greig, N., \& Luo, Y. (2017). Dopaminergic Neuron-Specific Deletion of p53 Gene Attenuates Methamphetamine Neurotoxicity. Neurotoxicity Research, 32(2), 218-230. doi: 10.1007/s12640-017-9723-z

Meda-Lara, R. M., Moreno-Jiménez, B., García, L. F., Palomera Chávez, A. \& Mariscal de Santiago, M. V. (2015). Validez factorial del NEO-FFI en una muestra mexicana: Propuesta de una versión reducida. Revista Mexicana de Psicología, 32(1), 57-67.

Müller, C. P., \& Kornhuber, J. (2017). Biological Evidence for Paradoxical Improvement of Psychiatric Disorder Symptoms by Addictive Drugs. Trends in Pharmacological Sciences, 38(6), 501-502. doi: 10.1016/j.tips.2017.03.005

Oh, J.-H., Hwang, J.-Y., Hong, S.-I., Ma, S.-X., Seo, J.-Y., Lee, S.Y., ... Jang, C.-G. (2016). The new designer drug buphedrone produces rewarding properties via dopamine D1 receptor activation. Addiction Biology, 23(1), 69-79. doi:10.1111/adb.12472

Ostrosky, F., Ardila, A. \& Roselli, M. (2013). Neuropsi: Evaluación Neuropsicológica Breve en español. México: Manual Moderno.

Ostrosky, F., Gomez, M. E., Matute, E., Rosselli, M., Ardila, A., \& Pineda D. (2019). Neuropsi: Atención y Memoria (3a. ed.). México: Manual Moderno.

Román, F., Santibáñez, P., \& Vinet, E. V. (2016). Use of the Depression Anxiety Stress Scales (DASS-21) as Screening Tests in Clinical Youngsters. Acta de Investigación Psicológica, 6(1), 2325-2336.

Shokoohi, M., Karamouzian, M., Sharifi, H., Rahimi-Movaghar, A., Carrico, A. W., Hosseini Hooshyar, S., \& Mirzazadeh, A. (2018). Crystal methanphetamine use and its correlates in women engaged in sex work in a developing country setting. Drug \& Alcohol Dependence, 185, 260-265. doi: 10.1016/j. drugalcdep.2017.12.025

Widiger, T. A., \& Costa Jr, P. T. (Eds.). (2013). Personality disorders and the five-factor model of personality. Washington, DC: American Psychological Association.

Wu, B., Lin, W., Wang, H., Abdullah, T., Wang, B., Su, Y., ... Lian, Q. (2018). Glucocorticoid receptor in rat nucleus accumbens: Its roles in propofol addictions. Neuroscience Letters, 662, 115121. doi: 10.1016/j.neulet.2017.10.011 